With the joint support of the National Natural Science Foundation of China (Grant No. 31570212, 31770228), the National Key Program of the Ministry of Science and Technology (Grant No. 2017FY100100) and the Talent Project of Yunnan (Grant No. 2015HBo92), the Kunming Institute of Botany surveyed for any remnant $L$. robusta in the southern Hengduan Mountain range during JuneAugust in 2018. Approximately 1,100 individuals in flower were discovered in three separate sites in grassy wetlands at an altitude of $1,730 \mathrm{~m}$. The total area of occupancy of the species is c. $800 \mathrm{~m}^{2}$. This suggests that it should be categorized as Critically Endangered on the IUCN Red List on the basis of criterion B2ab (i,ii,iii,v). Also, because of its restricted distribution, small population size, and habitat degradation, it should be included in the list of Plant Species with Extremely Small Populations in China (Ma et al., 2013, Biodiversity and Conservation, 22, 803-809). My survey and interviews with local people indicated that the main threats to this species are its small population size, the development of its wetland habitat for tourism in the last 15 years-leading to an increasing number of people picking the flowers for their ornamental value - and habitat loss because of road construction. Urgent and effective measures need to be taken to protect this species.

The Kunming Institute of Botany is now carrying out studies on the population genetics of L. robusta and its genetic relationships to other Lysimachia species of the southern Hengduan Mountains, to obtain a better understanding of the microevolution of this species. In collaboration with staff of nature reserves, I am planning to collect seeds of $L$. robusta for propagation and for potential restoration of the species in the wild. The southern Hengduan Mountains are currently managed as a nature reserve by the local government. However, more attention should be paid to wetlands in this region, because this is the only known natural habitat for the remnant $L$. robusta.

RoNG LI Key Laboratory for Plant Diversity and Biogeography of East Asia, Kunming Institute of Botany, Chinese Academy of Sciences, Kunming, China.E-mail lirong@mail.kib.ac.cn

\section{Rediscovery and conservation of the Critically Endangered Rhododendron griersonianum in Yunnan, China}

Rhododendron griersonianum Balf. f. et Forrest, belonging to subsection Griersoniana Davidian ex Chamb of the Ericaceae, is a Critically Endangered species on The Red List of Rhododendrons (Gibbs et al., 2011, Botanic Gardens Conservation International) and the Threatened
Species List of China's Higher Plants (Qin et al., 2017, Biodiversity Science, 25, 696-744). Circa 10\% of all registered Rhododendron cultivars have been created by hybridization with $R$. griersonianum. Despite its value for horticulture, the conservation of $R$. griersonianum has been afforded little attention. After its description in 1919 (Isaac Bayley \& Gorge, 1919, Notes from the Royal Botanic Garden, Edinburgh, 11, 69-71), Gorge Forrest collected the species again in 1924, 1925 and 1931. In $1964 \mathrm{Wu}$ Sugong of Kunming Institute of Botany, Chinese Academy of Sciences, collected one specimen, deposited in the Herbarium of Kunming Institute of Botany, Chinese Academy of Sciences. Since then the species had not, until now, been located again.

To secure up-to-date information on R. griersonianum we surveyed in its type locality during its flowering time in May 2015, but failed to find the species. In May 2016, however, with the help of local people, we surveyed again and three plants with flowers were found within an area of c. 100 $\mathrm{m}^{2}$ on steep rocky terrrain near farmland in Jietou, Tengchong County, west Yunnan. Following further searches we found four plants on a riverbed in Houqiao, c. $50 \mathrm{~km}$ from the first population. In July 2018 we found eight plants along a road in Guyong, c. $12 \mathrm{~km}$ from the first population and c. $42 \mathrm{~km}$ from the second population. We did not find seedlings in any of the three populations. Information obtained from interviews with local people and our field investigations indicated that the main threat to this species is the loss of suitable habitat as a result of human activities such as logging, farming, and reservoir and road construction.

Priority conservation action is required to prevent the extinction of this Critically Endangered and horticulturally important species. At Kunming Botanical Garden of Kunming Institute of Botany seedlings are being propagated, from both seeds and cuttings, for ex situ conservation, a local nature reserve has agreed to establish protection plots in the habitat of R. griersonianum for in situ conservation, and samples have been taken for phylogenetic research. In addition, further surveys to locate other potential populations are required. An integrated conservation action plan for the species is now being prepared.

Detuan Liu, Weibang Sun and Yongpeng Ma Yunnan Key Laboratory for Integrative Conservation of Plant Species with Extremely Small Populations, Kunming Botanical Garden, Kunming Institute of Botany, Chinese Academy of Sciences, Kunming, Yunnan, China E-mail mayongpeng@mail.kib.ac.cn

ZHENDONG FANG Shangri-la Alpine Botanical Garden, Shangri-la, Yunnan, China 